\title{
COMUNICAÇÃO
}

\section{FATORES QUE INFLUENCIAM A REAÇÃO DE SAPONIFICAÇÃO DOS CAROTENÓIDES PRESENTES NO URUCUM (Bixa orellana L.)}

\author{
Factors that influence saponification reaction in annatto (Bixa orellana L.) carotenoids
}

\author{
Pollyanna Ibrahim Silva ${ }^{1}$, Aline Manke Nachtigall², Paulo César Stringheta ${ }^{3}$
}

\begin{abstract}
RESUMO
Conduziu-se este trabalho, com o objetivo de estudar o efeito de diferentes concentrações de $\mathrm{KOH}(0,6 \%, 1 \%, 2 \%, 3 \%, 4 \%$ e 5\%) e temperatura de extração $\left(25^{\circ} \mathrm{C}, 50^{\circ} \mathrm{C}, 60^{\circ} \mathrm{C}\right.$ e $\left.70^{\circ} \mathrm{C}\right)$ na reação de saponificação dos principais pigmentos de urucum. $\mathrm{O}$ progresso da reação foi acompanhado pela quantificação do teor de bixina e de norbixina por meio de cromatografia líquida de alta eficiência (CLAE). A maior concentração de base propiciou a maior conversão de bixina em norbixina (KOH $5 \%$ - bixina: 0,44 g. $100 \mathrm{~g}$ ${ }^{1}$, norbixina: $1,43 \mathrm{~g} \cdot 100 \mathrm{~g}^{-1}$ ) e com a menor concentração não ocorreu conversão (KOH 0,6\% - bixina: 2,00 g. $100 \mathrm{~g}^{-1}$, norbixina: não detectada). A elevação da temperatura de extração esteve associada à redução do teor de bixina no meio e ao aumento do teor de norbixina ( $\mathrm{T}_{70^{\circ} \mathrm{C}}$ - bixina: $0,01 \mathrm{~g} .100 \mathrm{~g}^{-1}$, norbixina: $2,34 \mathrm{~g} .100 \mathrm{~g}^{-1} ; \mathrm{T}_{25^{\circ} \mathrm{C}}$ - bixina: $1,04 \mathrm{~g} .100 \mathrm{~g}^{-1}$, norbixina: $0,78 \mathrm{~g} \cdot 100 \mathrm{~g}^{-1}$ ). Sendo assim, para extração de bixina seria recomendado o uso de soluções alcalinas pouco concentradas, ao passo que para a obtenção do pigmento hidrossolúvel são aconselhadas concentrações mais elevadas, podendo estar associadas a aquecimento.
\end{abstract}

Termos para indexação: Bixina, Norbixina, concentração do álcali, temperatura de extração.

\section{ABSTRACT}

This research aimed to evaluate the effect of different $\mathrm{KOH}$ concentrations $(0.6 \%, 1 \%, 2 \%, 3 \%, 4 \%$ and $5 \%)$ and extraction temperature $\left(25^{\circ} \mathrm{C}, 50^{\circ} \mathrm{C}, 60^{\circ} \mathrm{C}\right.$ and $\left.70^{\circ} \mathrm{C}\right)$ on the progress of saponification reaction in annatto pigments. The progress of the reaction was monitored by quantification of bixin and norbixin using high performance liquid chromatography (HPLC). The highest alkali concentration made possible the maximum conversion of bixin into norbixin (KOH $5 \%$ - bixin: 0.44 g. $100 \mathrm{~g}^{-1}$, norbixin: 1.43

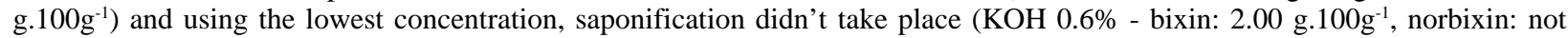
detected). The elevation of extraction temperature was associated to a decrease of bixin content and to an increase of norbixin content ( $\mathrm{T}_{70^{\circ} \mathrm{C}}$ - bixin: $0.01 \mathrm{~g} .100 \mathrm{~g}^{-1}$, norbixin: $2.34 \mathrm{~g} .100 \mathrm{~g}^{-1} ; \mathrm{T}_{25^{\circ} \mathrm{C}}$ - bixin: $1.04 \mathrm{~g} .100 \mathrm{~g}^{-1}$, norbixin: $\left.0.78 \mathrm{~g} .100 \mathrm{~g}^{-1}\right)$. Therefore, for the extraction of bixin is recommended to use alkaline solutions in low concentrations. To obtain the hydrossoluble pigment, higher concentrations are suggested, and they could be associated to heating.

Index terms: Bixin, Norbixin, concentration of alkali, extraction temperature.

(Recebido em 28 de abril de 2008 e aprovado em 12 de setembro de 2008)

Os corantes têm sido largamente utilizados como um meio de melhorar a aparência de alimentos, bebidas e cosméticos. O corante de urucum é utilizado extensivamente em suas várias formas, na indústria de alimentos, para tonalidades amarelas a vermelhas. O sal de norbixina, pigmento hidrossolúvel do urucum, é utilizado, principalmente, em queijos, sorvetes, derivados de cereais, confeitos, bebidas, molhos e salsichas, representando quase a totalidade do mercado de urucum (CONSTANT et al., 2002; COSTA \& CHAVES, 2005; KOUL et al., 2003; PIMENTEL \& STRINGHETA, 1999; SHUHAMA et al., 2003). A bixina, lipossolúvel, é usada em alimentos como margarinas, cremes vegetais, queijos e sorvetes (CONSTANT, 1999; ROSA, 2004).

A bixina é o pigmento natural da semente do urucum (Bixa orellana L.), representando mais de $80 \%$ de todos os carotenóides presentes, seguida da norbixina, encontrada em pequenas quantidades nas sementes, podendo ser

\footnotetext{
'Engenheira de Alimentos, Doutoranda em Ciência e Tecnologia de Alimentos - Universidade Federal de Viçosa - Departamento de Tecnologia de Alimentos/DTA - Universidade Federal de Viçosa/UFV - Universidade Federal de Viçosa, Departamento de Tecnologia de Alimentos - Laboratório de Corantes Naturais - Campus Universitário s/n - 365700-000 - Viçosa,MG - pollyannaibrahim@yhoo.com.br

'Bacharel em Química de Alimentos, Mestre em Ciência e Tecnologia Agroindustrial - Universidade Federal de Pelotas, Pós-doutorado em Ciência e Tecnologia de Alimentos da Universidade Federal de Viçosa - Departamento de Tecnologia de Alimentos/DTA - Universidade Federal de Viçosa/UFV Departamento de Tecnologia de Alimentos - Laboratório de Corantes Naturais - Campus Universitário s/n - 36570-000 - Viçosa,MG - anachtigall@gmail.com ${ }^{3}$ Engenheiro Agrônomo, Mestre em Ciência e Tecnologia de Alimentos - Universidade Federal de Viçosa, Doutor em Ciência de Alimentos - Universidade Estadual de Campinas, Professor Titular do Departamento de Tecnologia de Alimentos - Universidade Federal de Viçosa - Departamento de Tecnologia de Alimentos/DTA - Universidade Federal de Viçosa/UFV - Departamento de Tecnologia de Alimentos - Laboratório de Corantes Naturais - Campus Universitário s/n - 36570-000 - Viçosa,MG - pstringheta@yahoo.com.br
} 
produzida pela saponificação da bixina (BARETH et al., 2002; KOUL et al., 2003; RIOS \& MERCADANTE, 2004b; TOCCHINI \& MERCADANTE, 2001).

Ambas, bixina e norbixina, podem estar presentes em uma extração, tanto na forma cis como na trans (PRENTICE-HERNANDEZ et al., 1993; SCOTTER et al., 1998, 2001). A bixina, em condições alcalinas, pode sofrer saponificação e produzir o ácido dicarboxílico livre, denominado norbixina. Em excesso de álcali, o ácido dicarboxílico dissocia-se para formar seu sal, geralmente de potássio ou sódio, solúvel em água (PIMENTEL \& STRINGHETA, 1999).

A grande demanda dos extratos de urucum com características de elevada qualidade tem acentuado as deficiências dos processos comumente usados para produzir os corantes, incluindo riscos de degradação térmica dos extratos e de oxidação dos pigmentos. As quantidades relativas de bixina e seus produtos de isomerização e de degradação em preparações comerciais de urucum variam dentro de amplos limites, dependendo das condições de processamento (PRENTICEHERNANDEZ et al., 1992).

Com base no contexto da extração alcalina dos pigmentos de urucum, este trabalho foi realizado com o objetivo de avaliar a influência da concentração do álcali e da temperatura de extração na reação de saponificação dos carotenóides de urucum.

A matéria-prima empregada foi a semente de Bixa orellana L. cv. Embrapa 36/37.

Para avaliar o efeito da concentração do álcali na reação de saponificação dos pigmentos de urucum, utilizaram-se cinco diferentes concentrações da base $\mathrm{KOH}$ $(0,6 \%, 1 \%, 2 \%, 3 \%, 4 \%$ e $5 \%)$, durante uma hora.

Alíquotas de $50 \mathrm{~g}$ de sementes foram misturadas às diferentes concentrações de $\mathrm{KOH}$ na proporção de 1:6 (p/v). Após a adição da base as amostras foram submetidas à agitação constante a $108 \mathrm{rpm}$, com auxílio de um banho de água a $50 \pm 1^{\circ} \mathrm{C}$ (Nova Ética, modelo 304/D, São Paulo, Brasil), ao abrigo da luz.

Para avaliar o efeito da temperatura na reação de saponificação dos pigmentos de urucum, as extrações foram conduzidas a $25^{\circ} \mathrm{C}, 50^{\circ} \mathrm{C}, 60^{\circ} \mathrm{C}$ e $70^{\circ} \mathrm{C}\left( \pm 1^{\circ} \mathrm{C}\right)$. Com base nos resultados do ensaio anterior, empregou-se $\mathrm{KOH}$ na concentração de $4 \%$ como o álcali $(1: 6, \mathrm{p} / \mathrm{v})$. As amostras foram submetidas à agitação (108 rpm), fazendo uso de um banho de água, ao abrigo da luz, nas temperaturas avaliadas, durante uma hora.

Após as extrações, alíquotas dos extratos foram diluídas em uma mistura de água:etanol 92,8\% (2:1).

Para a identificação e quantificação dos pigmentos de urucum, empregou-se CLAE, com fase móvel de acetonitrila: ácido acético 5\% (75:25), baseado em Bareth et al. (2002). Foi utilizado cromatógrafo líquido da marca Shimadzu, modelo LC-10 AD VP (Kyoto, Japan), detector UV-Visível e integrador-processador Shimadzu SCL-10A VP, com duas bombas e sistema manual de injeção. As amostras, após diluídas na mistura de água:etanol 92,8\% (2:1), foram todas filtradas em membrana Milipore ${ }^{\mathrm{o}}$ de nylon, com diâmetro de poro de 0,2 mm, e injetadas no sistema CLAE.

Utilizou-se coluna de fase reversa Lichrospher 100 RP-18 (25 cm ` 4,0 mm; 5 ìm) marca Merck (Darmstadt, Germany), protegida por uma pré-coluna CLC G-ODS, da marca Shimadzu (Kyoto, Japan). A vazão da fase móvel foi de 1,0 mL. $\mathrm{min}^{-1}$ e os espectros foram adquiridos no comprimento de onda de $460 \mathrm{~nm}$. O volume de amostra injetado foi de $50 \mu \mathrm{L}$.

A quantificação foi feita pelo método do padrão externo. Os padrões de bixina $(97,8 \%)$ e norbixina $(91,3 \%)$ foram preparados no laboratório, a partir de sementes de urucum. As curvas analíticas (área versus concentração) para bixina e norbixina foram obtidas por meio da análise de soluções dos padrões com concentrações de 50 a 500 g. $\mathrm{L}^{-1}$, obtendo-se coeficientes de determinação $\left(\mathrm{R}^{2}\right)$ de 0,986 e 0,981 , respectivamente.

O experimento foi realizado de forma que as condições do segundo ensaio foram definidas a partir da melhor condição determinada no primeiro ensaio. As extrações e determinações foram efetuadas com três repetições.

Na Figura 1, é possível visualizar o efeito da concentração de base na conversão de bixina em norbixina.

Ao observar a Figura 1, percebe-se que a conversão de bixina em norbixina ocorreu de forma crescente e proporcional ao aumento da concentração da base extratora. O comportamento citado é confirmado ao avaliar a Tabela 1, onde se observa que a extração com KOH 0,6\% a $50^{\circ} \mathrm{C}$ por uma hora não foi capaz de promover a saponificação do extrato, ao passo que ao empregar $\mathrm{KOH}$ $5 \%$ ocorreu predominância de norbixina no mesmo $(84,3 \%)$.

A saponificação parcial do grupo éster da bixina em sua forma hidrossolúvel, ou seja, o sal da norbixina, obtida em concentrações de $\mathrm{KOH}$ acima de $1 \%$, mostra dependência em relação à concentração do álcali. Fato este confirmado em estudos de Silva (2007), onde o autor verificou que a reação de saponificação da bixina em norbixina ocorre em valores de $\mathrm{pH}$ acima de 10 e é dependente, em grande parte, da concentração e da natureza do álcali, e em menor extensão, do tempo e da temperatura de extração. Rios \& Mercadante (2004a), ao estudar a influência do tempo, da temperatura e da concentração de $\mathrm{KOH}$ na saponificação dos pigmentos de urucum, utilizando metodologia de superfície de respostas, verificaram que a concentração de $\mathrm{KOH}$ foi o fator mais importante na saponificação, com a temperatura exercendo menor influência. 


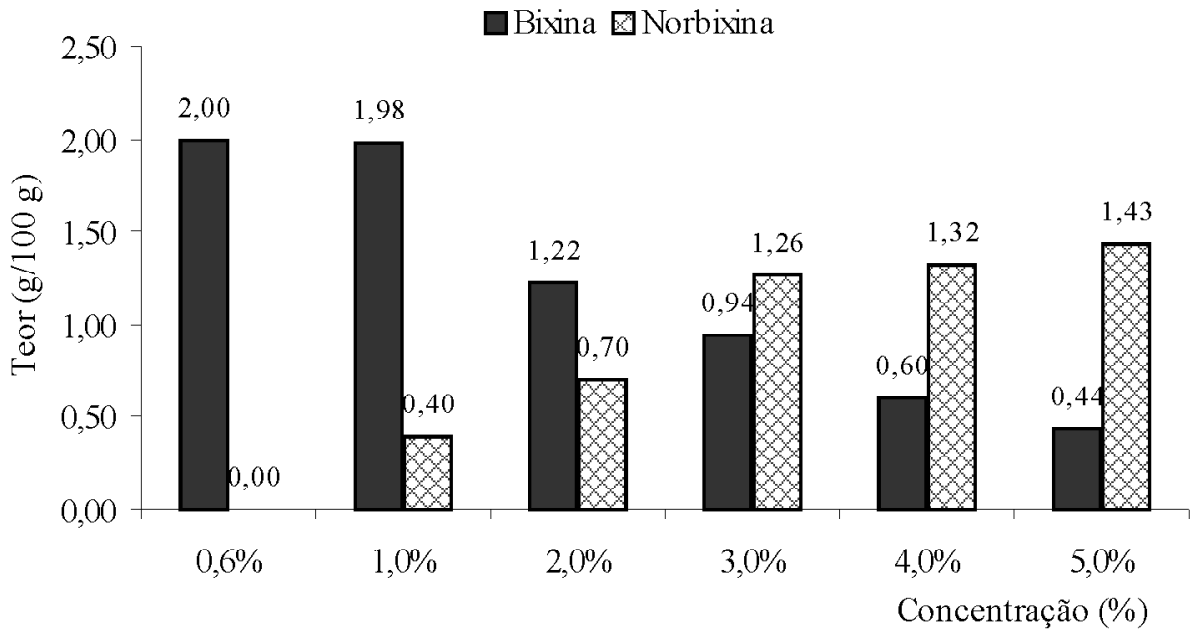

Figura 1 - Teores de bixina e norbixina, obtidos por CLAE, para os extratos obtidos com $\mathrm{KOH}$ a $50^{\circ} \mathrm{C}$, nas concentrações de 0,6 a $5 \%$, durante $1 \mathrm{~h}$ de extração.

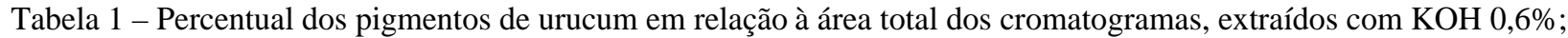
$1 \% ; 2 \% ; 3 \% ; 4 \%$ e $5 \%$, durante $1 \mathrm{~h}$ a $50^{\circ} \mathrm{C}$.

\begin{tabular}{ccc}
\hline Tratamento & Bixina $(\%)$ & Sal de norbixina $(\%)$ \\
\hline $\mathrm{KOH} 0,6 \% 50^{\circ} \mathrm{C}$ & 97,1 & nd \\
$\mathrm{KOH} 1 \% 50^{\circ} \mathrm{C}$ & 79,4 & 12,7 \\
$\mathrm{KOH} \% 50^{\circ} \mathrm{C}$ & 62,9 & 30,6 \\
$\mathrm{KOH} 3 \% 50^{\circ} \mathrm{C}$ & 42,5 & 51,2 \\
$\mathrm{KOH} 4 \% 50^{\circ} \mathrm{C}$ & 18,7 & 74,5 \\
$\mathrm{KOH} 5 \% 50^{\circ} \mathrm{C}$ & 9,2 & 84,3 \\
\hline
\end{tabular}

nd- não detectado.

Póvoa (1992), ao empregar KOH 0,5\% na extração de pigmentos das sementes de urucum, obteve aproximadamente $98 \%$ de pigmentos. Estes dados corroboram com os encontrados para a extração dos pigmentos de urucum com $\mathrm{KOH} 0,6 \%$.

Prentice-Hernandez et al. (1992), ao avaliarem o efeito da concentração de $\mathrm{NaOH}$ sob o percentual de bixina e norbixina produzidas, observaram que as soluções de $\mathrm{NaOH} 0,2$ e $0,3 \%$ não proporcionaram a formação de norbixina. A partir das concentrações de 0,4 a $0,6 \%$, foi verificada a saponificação do grupo éster, formando a norbixina dentro de uma reação considerada irreversível. Estes resultados discordam em parte dos encontrados no presente trabalho, uma vez que ao empregar uma base forte como KOH a $0,6 \%$ a reação de saponificação não ocorreu. No entanto, confirmou-se o fato de que a reação de hidrólise do grupamento éster da bixina é dependente da concentração da base e que é necessária uma concentração mínima para que a reação ocorra. Os autores verificaram ainda que, ao se utilizar bases com pH elevado há um progressivo aumento da concentração de trans-bixina no meio. Segundo Silva (2007), de acordo com o solvente e com o pH da extração utilizados, podem coexistir no meio as várias formas dos pigmentos.

Pimentel(1995), ao avaliar parâmetros da extração de pigmentos de sementes de urucum utilizando $\mathrm{KOH}$, verificou que a melhor condição para a extração de norbixina foi $\mathrm{KOH} 0,1 \mathrm{~N}$ a $50^{\circ} \mathrm{C}$, na proporção semente:solvente de 1:6 (p/v), encontrando um teor médio de $1,75 \%$. Estes dados discordam dos encontrados no presente trabalho, uma vez que ao empregar o $\mathrm{KOH} 0,6 \%$, que equivale à solução $0,1 \mathrm{~N}$ estudada pelo autor, verificouse a ausência da conversão de bixina em norbixina ou seu sal (Tabela 1).

Para avaliar o efeito de diferentes temperaturas na saponificação dos pigmentos de urucum, selecionou-se 
como solução extratora o $\mathrm{KOH} \mathrm{4 \% ,} \mathrm{uma} \mathrm{vez} \mathrm{que} \mathrm{nesta}$ concentração verificou-se uma conversão parcial da bixina em norbixina e, de acordo com relatos da literatura (PRENTICEHERNANDEZ et al., 1992; SILVA, 2007), nesta concentração podem ocorrer reações de degradação e isomerização.

$\mathrm{Na}$ Figura 2 constatam-se teores de bixina e norbixina dos extratos produzidos pelo solvente $\mathrm{KOH} 4 \%$, durante uma hora de extração, às temperaturas de $25^{\circ} \mathrm{C}$, $50^{\circ} \mathrm{C}, 60^{\circ} \mathrm{C}$ e $70^{\circ} \mathrm{C}$.

$\mathrm{Na}$ temperatura de $25^{\circ} \mathrm{C}$, o $\mathrm{KOH} 4 \%$ promoveu a saponificação parcial da bixina em norbixina em uma hora de extração. Ao elevar a temperatura de extração para $70^{\circ} \mathrm{C}$ verificou-se a conversão total de bixina em norbixina. $\mathrm{Na}$ Figura 3, pode-se visualizar o efeito da temperatura no andamento da reação, e observar que a $25^{\circ} \mathrm{C}$ o teor de norbixina correspondeu a $37,1 \%$ em relação ao total de carotenóides extraídos, aumentando para $88,2 \%$ a $60^{\circ} \mathrm{C}$ e a $92,4 \%$ quando submetido à temperatura de $70^{\circ} \mathrm{C}$.

Prentice-Hernandez et al. (1993) observaram que em condições de extração com pH superior a 11 ocorre predominantemente a isomerização cis-trans da bixina, e não a hidrólise da bixina produzindo a norbixina, sendo que a última só ocorre mediante aplicação de calor. No presente trabalho, ao se utilizar $\mathrm{KOH} 4 \%$, que apresenta um $\mathrm{pH}$ acima de 13, houve a presença de picos minoritários nos cromatogramas, não identificados, indicando possível formação de isômeros (Figura 3). Entretanto, de forma mais marcante, foi verificada hidrólise da bixina formando norbixina, mesmo à temperatura ambiente e em temperaturas mais altas como $70^{\circ} \mathrm{C}$.
Verificou-se, portanto, que a saponificação completa dos pigmentos de urucum, nas condições estudadas, se deu utilizando $\mathrm{KOH} 4 \%$ durante uma hora a $70^{\circ} \mathrm{C}$. Entretanto, Rios \& Mercadante (2004b) observaram que a conversão de bixina a norbixina durante a saponificação foi de $100 \%$ apenas quando se utilizou $\mathrm{KOH}$ metanólico 55\%, durante 20 minutos a $50^{\circ} \mathrm{C}$, sem formação de isômeros adicionais. Observaram também que independente da concentração de $\mathrm{KOH}$, notou-se maior presença de isômeros quando a saponificação foi realizada por longo período à temperatura ambiente do que em um tempo curto com aquecimento.

Em geral, quanto mais concentrada a base, mais completa será a conversão da bixina em norbixina e seu sal. Tempos de extração mais prolongados podem proporcionar maior conversão da bixina em norbixina, porém podem também ocasionar degradação destes compostos. Quando temperaturas mais elevadas são utilizadas, há um aumento no rendimento de extração dos pigmentos, porém com pouco incremento na reação de hidrólise da bixina em norbixina, ao se utilizar soluções alcalinas pouco concentradas.

De acordo com Silva (2007), o que se observa de forma mais marcante é a isomerização cis-trans da bixina e da norbixina, sendo as configurações trans consideradas mais estáveis. Segundo Henry (1996) e Scotter et al. (1998), na extração com solvente alcalino empregando temperaturas acima de $70^{\circ} \mathrm{C}$ há tendência à formação de isômeros trans da norbixina via reação de isomerização termicamente direcionada. A isomerização e a degradação do pigmento podem ocorrer simultaneamente.

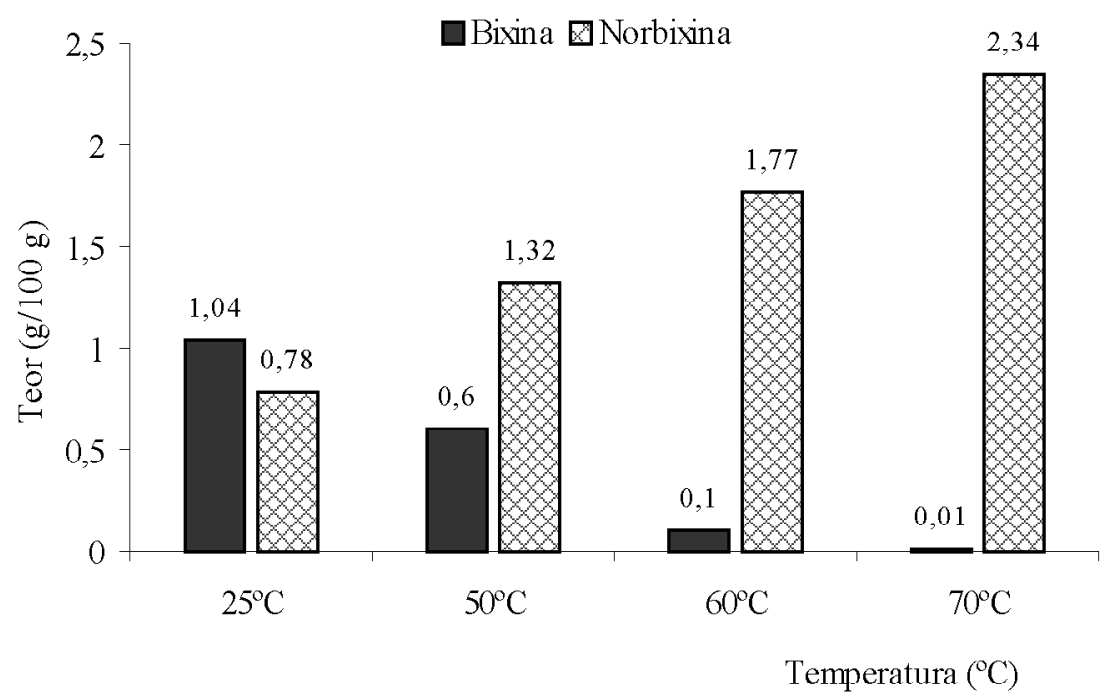

Figura 2 - Teores de bixina e norbixina, obtidos por CLAE, dos extratos obtidos com KOH 4\%, durante 1 h de extração, a $25^{\circ} \mathrm{C}, 50^{\circ} \mathrm{C}, 60^{\circ} \mathrm{C}$ e $70^{\circ} \mathrm{C}$. 

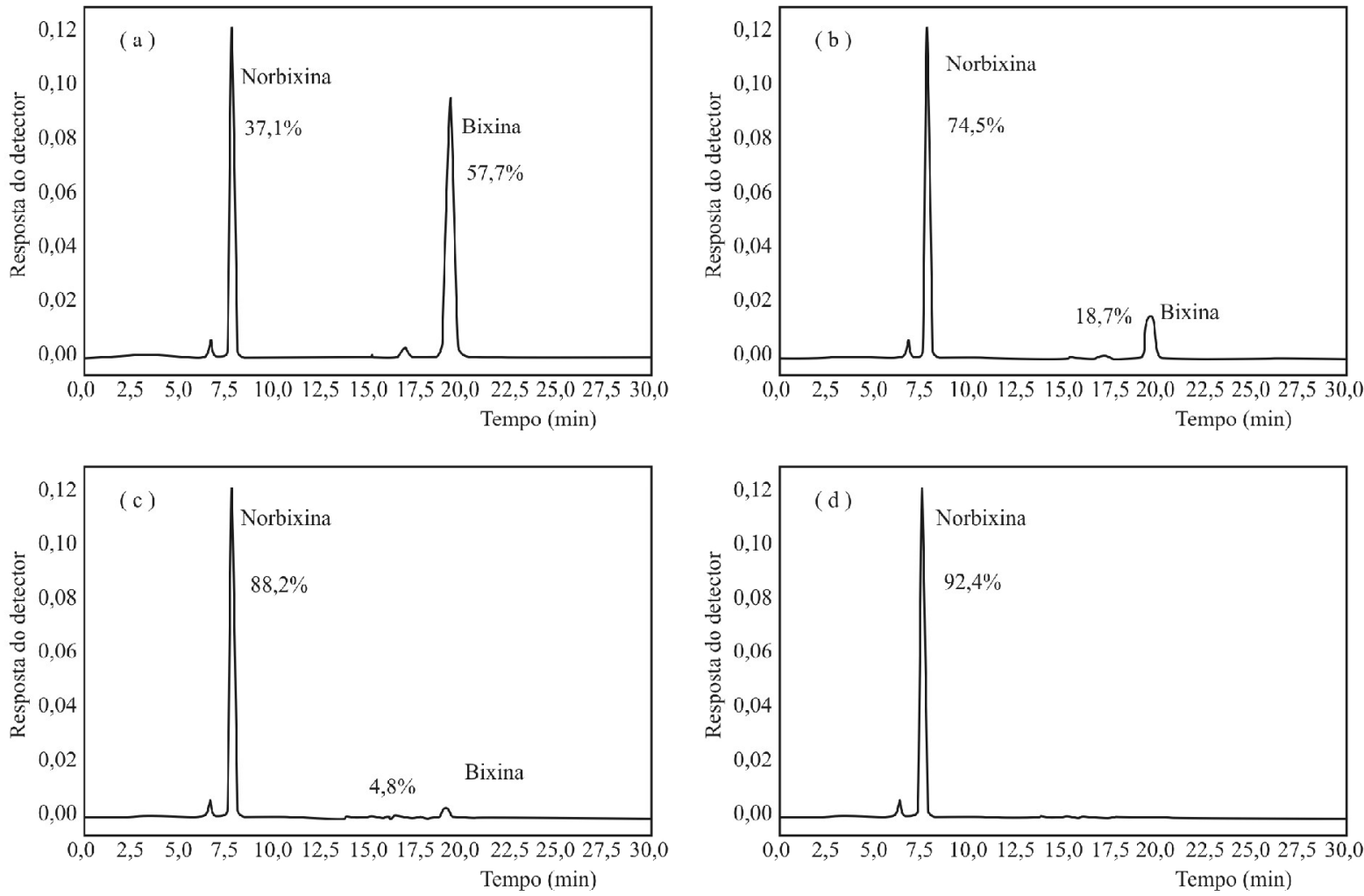

Figura 3 - Cromatogramas, obtidos por CLAE, dos extratos produzidos com KOH 4\%, durante uma hora de extração, a $25^{\circ} \mathrm{C}(\mathrm{a}), 50^{\circ} \mathrm{C}(\mathrm{b}), 60^{\circ} \mathrm{C}$ (c) e $70^{\circ} \mathrm{C}(\mathrm{d})$. Condições cromatográficas: vide texto.

Araújo (2001) afirma que, apesar de apresentar características inerentes aos carotenóides, de modo geral, os pigmentos de urucum podem ser considerados bastante estáveis, principalmente quando comparados com outros grupos de corantes naturais. A bixina é sensível às variações de $\mathrm{pH}$, tendo a coloração alterada do amarelo alaranjado para o rosa fraco. Entretanto, em $\mathrm{pH}$ reduzido apresenta estabilidade térmica satisfatória a temperaturas abaixo de $100^{\circ} \mathrm{C}$.

A reação de hidrólise da bixina para formar a norbixina é dependente da concentração do solvente alcalino utilizado e da temperatura empregada no processo. Tanto o aumento da concentração das soluções de $\mathrm{KOH}$, quanto a elevação da temperatura de extração levaram a uma maior conversão da bixina em norbixina.

A melhor condição para a obtenção da bixina pode ser a associação de reduzida concentração de álcali a baixas temperaturas. Já para a produção do pigmento hidrossolúvel pode-se efetuar a extração empregando $\mathrm{KOH} 4 \%$ a $70^{\circ} \mathrm{C}$, sem que ocorra degradação do pigmento formado.

São necessárias pesquisas adicionais visando a avaliar a interação entre as variáveis tempo, temperatura e natureza do álcali (diferentes bases em meio aquoso ou alcoólico), envolvidas na saponificação de bixina em norbixina, de forma a otimizar o andamento desta reação e a produção dos compostos de interesse.

\section{AGRADECIMENTOS}

Os autores agradecem à Fundação de Amparo à Pesquisa do Estado de Minas Gerais (FAPEMIG) pela bolsa de pós-doutorado concedida, e ao Conselho Nacional de Desenvolvimento Científico e Tecnológico (CNPq) pelo suporte financeiro.

\section{REFERÊNCIAS BIBLIOGRÁFICAS}

ARAÚJO, J. M. A. Química de alimentos: teoria e prática. 2. ed. Viçosa: UFV, 2001. 416 p.

BARETH, A.; STROHMAR, W.; KITZELMANN, E. HPLC and spectrophotometric determination of annatto in cheese. European Food Research and Technology, Heildeberg, v. 215, n. 4, p. 359-364, 2002. 
CONSTANT, P. B. L. Microencapsulamento de Bixina: agentes encapsulantes, avaliação da qualidade e aplicações. 1999. 136 f. Dissertação (Mestrado em Ciência e Tecnologia de Alimentos) - Universidade Federal de Viçosa, Viçosa, 1999.

CONSTANT, P. B. L.; STRINGHETA, P. C.; SANDI, D. Corantes alimentícios. Boletim do Ceppa, Curitiba, v. 20, n. 2, p. 203-220, 2002.

COSTA, C. L. S.; CHAVES, M. H. Extração de pigmentos das sementes de Bixa orellana L.: uma alternativa para disciplinas experimentais de química orgânica. Química Nova, São Paulo, v. 28, n. 1, p. 149-152, 2005.

HENRY, B. S. Natural food colours. In: HENDRY, G. A. F.; HOUGHTON, J. D. Natural Food Colorants. 2. ed. Glasgow: Blackie Academic \& Professional, 1996. p. 4079 .

KOUL, V. K.; KOUL, S.; TIKOO, C. L. Process optimization for extraction and purification of bixin from annatto. Indian Journal of Chemical Technology, New Delhi, v. 10, p. 545-547, 2003.

PIMENTEL, F. A. Avaliação de métodos de obtenção e de estabilidade de pigmentos de sementes de urucum. 1995. 132 f. Dissertação (Mestrado em Ciência e Tecnologia de Alimentos) - Universidade Federal de Viçosa, Viçosa, 1995.

PIMENTEL, F. A.; STRINGHETA, P. C. Produção de corantes de urucum em pó, por meio de precipitação ácida, a partir de extratos obtidos em diferentes soluções extratoras. Revista Brasileira de Corantes Naturais, Viçosa, v. 3, n. 1, p. 53-57, 1999.

PÓVOA, M. E. B. Extração do corante de urucum (Bixa orellana L.) com diversos solventes. Revista Brasileira de Corantes Naturais, Viçosa, v. 1, n. 1, p. 153-157, 1992.

PRENTICE-HERNANDEZ, C.; RUSIG, O.; CARVALHO, P. R. N. Efeito do $\mathrm{pH}$ na quantidade de bixina obtida em extratos alcalinos de urucum (Bixa orellana L.). Revista Brasileira de Corantes Naturais, Viçosa, v. 1, n. 1, p. 182-187, 1992.

PRENTICE-HERNANDEZ, C.; RUSIG, O.; CARVALHO, P. R. N. Influência do tempo de aquecimento na degradação térmica de bixina em extratos alcalinos de urucum (Bixa orellana L.). Arquivos de Biologia e Tecnologia, Curitiba, v. 36, n. 4, p. 819-828, 1993.

RIOS, A. de O.; MERCADANTE, A. Z. Novel method for the determination of added annatto colour in extruded corn snack products. Food Additives and Contaminants, London, v. 21, n. 2, p. 125-133, 2004a.

RIOS, A. de O.; MERCADANTE, A. Z. Otimização das condições para obtenção de padrão de bixina e das etapas de extração e saponificação para quantificação de bixina em "snacks" extrusados por CLAE. Alimentos e Nutrição, Araraquara, v. 15, n. 3, p. 203-213, 2004 b.

ROSA, L. C. C. Corantes naturais em alimentos: preferência do consumidor e aplicação industrial. 2004. 104 f. Dissertação (Mestrado em Ciência e Tecnologia de Alimentos) - Universidade Federal de Viçosa, Viçosa, 2004.

SCOTTER, M. J.; CASTLE, L.; APPLETON, G. P. Kinetics and yields for the formation of coloured and aromatic thermal degradation products of annatto in foods. Food Chemistry, Kidlington, v. 74, n. 3, p. 365-375, 2001.

SCOTTER, M. J.; WILSON, L. A.; APPLETON, G. P.; CASTLE, L. Analysis of annatto (Bixa orellana) food coloring formulations: 1 . determination of coloring components and colored thermal degradation products by high-performance liquid chromatography with photodiode array detection. Journal of Agricultural and Food Chemistry, Washington, v. 46, n. 3, p. 1031-1038, 1998.

SHUHAMA, I. K.; AGUIAR, M. L.; OLIVEIRA, W. P.; FREITAS, L. A. P. Experimental production of annatto powders in spouted bed dryer. Journal of Food Engineering, Kidlington, v. 59, n. 1, p. 93-97, 2003.

SILVA, P. I. Métodos de extração e caracterização de bixina e norbixina em sementes de urucum (Bixa orellana L.). 2007. 145 f. Dissertação (Mestrado em Ciência e Tecnologia de Alimentos) - Universidade Federal de Viçosa, Viçosa, 2007.

TOCCHINI, L.; MERCADANTE, A. Z. Extração e determinação, por CLAE, de bixina e norbixina em coloríficos. Ciência e Tecnologia de Alimentos, Campinas, v. 21, n. 3, p. 310-313, 2001. 